\title{
Effects of a functional appliance on masticatory muscles of young adults suffering from muscle-related temporomandibular disorders
}

\author{
T. CASTROFLORIO, F. TALPONE, A. DEREGIBUS, M. G. PIANCINO \\ \& P. B R A C C O Specialisation School of Orthodontics, Department of Biomedical Sciences and Human Oncology, School of Dentistry, \\ Turin University, Turin, Italy
}

SUMMARY The aim of this study was to investigate the effects of an original orthodontic functional appliance [function generating bite for deep bite correction (FGB-D)] on masticatory muscle activity in subjects suffering from muscle-related temporomandibular disorders (TMD). Electromyographic (EMG) analysis was performed on 33 young adults (nine men, 24 women) to evaluate the contractile symmetry of the right and left masseter and anterior temporalis muscles. The subjects were divided into three groups: a muscle-related TMD group requiring orthodontic treatment for deep bite correction (three men, eight women) and treated with FGB-D; a muscle-related TMD group not requiring orthodontic treatment (three men, eight women) and treated with a Michigan occlusal splint; and a TMD-free group (three men, eight women) as a control group. Records were made by surface EMG of maximum voluntary teeth clenching, with and without the functional appliance or occlusal splint in place, before and after 12 months of therapy. A torque index was derived from the surface EMG recordings to estimate lateral displacement of the mandible. The results show that the FGB-D corrects the torque index and thus the lateral displacement of the mandible. KEYWORDS: surface electromyography, functional appliance, torque index, masticatory muscles

Accepted for publication 28 March 2003

\section{Introduction}

The lack of standardized diagnostic criteria was a critical obstacle towards a further understanding of temporomandibular disorders (TMD). A significant contribution was made by Dworkin and Le Resche (1) with the introduction of a set of research diagnostic criteria for TMD, labelled 'RDC/TMD'.

According to RDC, TMD can be classified into three major groups: (1) muscle conditions, (2) disc displacements, and (3) arthralgia, arthritis, and arthrosis, on the basis of a simple physical examination and case history.

The muscle-related conditions represent the most prevalent conditions among the TMD, with at least $50 \%$ of cases falling into this category (2). Pain or ache in the jaw, temples, face, pre-auricular area, or inside the ear at rest or during function combined with tenderness to palpation of at least three of 20 palpation sites, with at least one ipsilateral to the complaint pain, must be present to be diagnosed as muscle-related TMD (1).

Quantitative surface electromyography (sEMG) of masticatory muscles is a reliable technique for the assessment of TMD patients $(3,4)$. The study of the electrical performance of masticatory muscles in muscle-related TMD patients, during predefined tasks, can aid in the development of diagnostic criteria and may allow the monitoring of the progression of the disease and the effects of therapy.

Even if occlusion as an etiologic factor of TMD is still under debate, significant associations have been found between TMD and several kinds of malocclusion (5-8). The treatment of TMD by interocclusal devices followed 
two paths: occlusal splints and functional orthopedic appliances. Occlusal splints eliminate the potential triggering factor but do not modify the occlusal discrepancies.

Functional appliances help in the movement of the teeth, with the achievement of good facial muscle function (9-12). This suggested the use of an original functional appliance called function generating bite for deep bite correction (FGB-D) in the treatment of muscle-related TMD patients requiring orthodontic treatment. This kind of appliance with metallic bite planes acts simultaneously as an orthodontic corrector $(9,13-16)$ and as an occlusal splint.

To evaluate the effects of the FGB-D on the masticatory muscles, the EMG activity of left and right masseter and temporalis anterior muscles during clenching has been investigated in a group of musclerelated TMD subjects requiring orthodontic treatment, just before the therapy and after 12 months of therapy.

\section{Materials and methods}

Subjects

Three groups of 11 subjects each (three men, eight women in each group) were selected for this study. Two groups were selected from a group of TMD patients presenting to the Orthodontics and Gnathology Unit, Turin University, where orthodontic and TMD patients are treated. The first group ( $\mathrm{F}$ - functional, mean age $25 \cdot 4 \pm 6.3$ years) was suffering from muscle-related TMD requiring orthodontic treatment for deep bite correction. This group was treated with FGB-D. The second group ( $\mathrm{S}-$ splint, mean age $27 \cdot 2 \pm 4 \cdot 2$ years) was suffering from muscle-related TMD not requiring orthodontic treatment. This group was treated with a Michigan occlusal splint (17). Inclusive criteria for both group $\mathrm{F}$ and $\mathrm{S}$ were: (1) diagnosis of muscular conditions, according to Dworkin and Le Resche (1); (2) absence of any kind of fixed or removable prosthetic restorations; (3) absence of periodontal disease; and (4) presence of all teeth (with the exception of the third molars). During the 12 months of therapy the patients were treated by expert operators.

A control group (C - control, mean age $26 \cdot 3 \pm 3 \cdot 1$ years) of healthy subjects was selected from the students of the Orthodontics Specialization School of Turin University. Furthermore these subjects had (1) any kind of fixed or removable prosthetic restorations,
(2) no periodontal disease, (3) presence of all teeth (with the exception of the third molars), and (4) any kind of orthodontic or occlusal therapy in the previous 12 months. These subjects did not wear any appliance or splint during the period considered in this study. All subjects gave their written informed consent.

\section{Instrumentation}

The superficial bundle of the right and left masseter muscles (MM) and the right and left temporalis anterior muscles were studied. Disposable silver/silver chloride bipolar surface electrodes DUO-TRODE*, diameter $10 \mathrm{~mm}$ and inter-electrode distance of $21 \mathrm{~mm}$, were positioned on the muscle bellies parallel to the muscle fibres: for the masseter the belly was palpated during clenching and the electrodes were fixed parallel to the fibres $2.5 \mathrm{~cm}$ above the mandibular angle to avoid influence from the end-plate location (18); for the temporalis anterior the belly was palpated during clenching and the electrodes were fixed along the anterior margin of the muscle $2 \mathrm{~cm}$ above the zygomatic arch (19). A disposable ground electrode was fixed to the right sternocleidomastoid muscle.

Before electrode placement, the skin was carefully cleansed with abrasive paste to reduce electrode impedance, and recordings were made after 5 min to allow absorption of the conductive gel by the skin.

Surface EMG recordings were obtained from four channels of the eight-channel electromyograph FREELY ${ }^{\dagger}$. The EMG signal was amplified, digitized and digitally filtered. The instrument was interfaced with a personal computer (PC) which displayed the data graphically and stored them on magnetic media for later analysis (EMA software ${ }^{\dagger}$ ). The signals were assessed as the root mean square (RMS) of the amplitude.

\section{Measurement}

Surface EMG recordings were obtained during two sessions: before treatment began (T0) and after 12 months of active treatment, with and without $\left(\mathrm{Tl}_{\mathrm{W}}\right.$ and $\mathrm{Tl}_{\mathrm{wo}}$, respectively) appliance/splint in place. The control group was monitored at the same time.

The subjects sat upright without head support; they fixed their eyes on a target (a green rectangle

\footnotetext{
*Myotronics Inc, Tukwila, WA, USA.

${ }^{\dagger}$ DeGoetzen spa, Olgiate Olona, VA, Italy.
} 
$30 \mathrm{~cm} \times 5 \mathrm{~cm}$ ) in front of them at $90 \mathrm{~cm}$. Testing was performed in a silent and comfortable environment.

In the first session (T0) the subjects were asked to perform maximum voluntary clenching (MVC) ('clench as hard as possible') in the intercuspal position. In the second session, after 12 months, they performed maximum voluntary clenching with FGB-D or splint in place $\left(\mathrm{Tl}_{\mathrm{w}}\right)$ and then, after a rest of $30 \mathrm{~min}$ without removing the electrodes and without any appliance or splint in place, in the intercuspal position $\left(\mathrm{Tl}_{\mathrm{wo}}\right)$.

At each session, a first recording for the standardization of EMG potentials was made as follows. In each subject, two cotton rolls (diameter $10 \mathrm{~mm}$ ) were positioned on the mandibular teeth (second premolar and molars), and the maximum voluntary clench was recorded. For each muscle, the maximum potential was found and set at $100 \%$ and all further EMG potentials were expressed as a percentage of this value (units, $\mu \mathrm{V}$ / $\mu \mathrm{V} \%$ ) (20). To avoid muscle fatigue, a rest period of $3 \mathrm{~min}$ was allowed between recordings. Each test was repeated three times at each session. Each recording lasted for $5 \mathrm{~s}$ followed by a rest period of $3 \mathrm{~min}$.

\section{Function generating bite for deep bite correction}

The FGB-D was designed over 20 years ago $(9,13)$; its orthodontic characteristics will not be discussed here, as they have been described in detail elsewhere (14-16). However, the most important characteristic, for the FGB-D (Fig. 1), is the presence of anterior and posterior metallic bite planes, which disengage the mandible from occlusion.

\section{Statistical calculations}

The direction of the forces of the masseter and temporalis anterior muscles may produce a lateral deviation of the mandible. To evaluate this effect a torque index, was calculated from the averaged sEMG potentials as proposed by Ferrario et al. (21):

$$
\text { Torque index }=\frac{(\text { TAR }+M M L)-(T A L+M M R)}{(T A R+M M L)+(T A L+M M R)} \%,
$$

where TAR and TAL are the temporalis anterior muscles RMS values (right and left, respectively) and MMR and MML are the RMS values of masseter muscles (right and left, respectively).

This index ranges from $-100 \%$ to $+100 \%$ : positive values indicating a stronger right-side resultant force, and negative values a stronger left-side resultant force. According to Ferrario et al. (21), during MVC the normal value of the torque index is $9 \cdot 47 \pm 7 \cdot 19 \%$. Indices were calculated automatically for each test by EMA software.

The mean torque index was calculated from the three tests for each subject at each session. Mean values and standard deviations were calculated for the torque indices, and also for the positive and negative values taken separately. Torque indices before and after therapy were compared using Student's $t$-test for paired data. The data were also analysed using one-way repeated measurement analysis of variance (ANOva), followed by post hoc Tukey-Kramer multiple comparisons test when required.

\section{Results}

Absolute mean values and standard deviations of torque indices are reported in Table 1. Results of Student's $t$-test are reported in Table 2.

In the group $\mathrm{F}$, all subjects showed an above-normal torque index at $\mathrm{T} 0$. The values decreased at $\mathrm{T} \mathrm{l}_{\mathrm{wo}}$, after 12 months of therapy with FGB-D (Fig. 2$)(P<0.05)$. A further reduction (Fig. 3) was observed at $\mathrm{Tl}_{\mathrm{w}}$ $(P<0.05)$. At the beginning of therapy, subjects in group $\mathrm{S}$ had a normal torque index. The value was

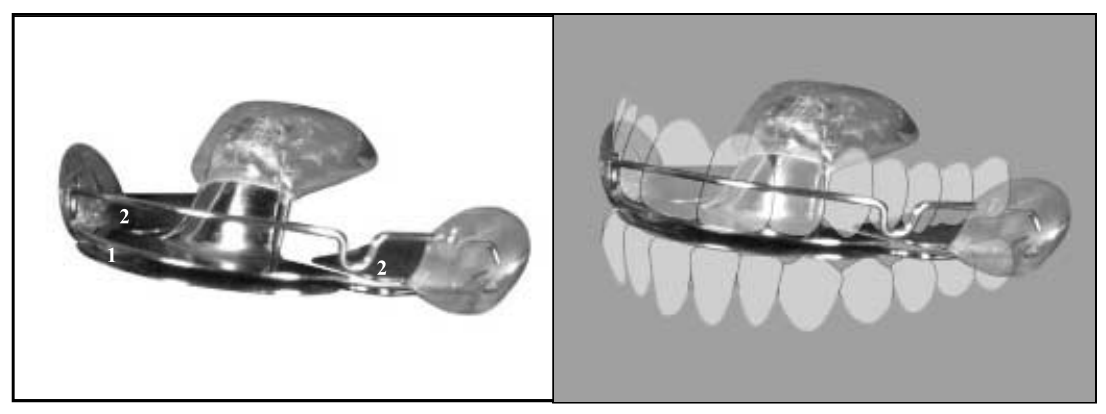

Fig. 1. The appliance and a schematic representation of the FGB-D in place. (1) anterior metallic bite planes; (2) posterior metallic bite plane. 
Table 1. Torque index (TOR): mean values and standard deviation at $\mathrm{T} 0, \mathrm{Tl}_{\mathrm{wo}}$ and $\mathrm{T} 1_{\mathrm{w}}$

\begin{tabular}{|c|c|c|c|c|c|c|}
\hline & \multicolumn{2}{|l|}{ T0 } & \multicolumn{2}{|l|}{$\mathrm{Tl}_{\text {wo }}$} & \multicolumn{2}{|l|}{$\mathrm{T} 1_{\mathrm{w}}$} \\
\hline & Mean & s.d. & Mean & s.d. & Mean & s.d. \\
\hline Group F & $52 \cdot 27$ & $14 \cdot 24$ & $17 \cdot 05$ & $14 \cdot 96$ & $6 \cdot 06$ & 6.58 \\
\hline Group S & 10.53 & $7 \cdot 18$ & $12 \cdot 92$ & $11 \cdot 84$ & $9 \cdot 95$ & $12 \cdot 42$ \\
\hline Group C & $6 \cdot 01$ & $1 \cdot 13$ & $5 \cdot 66$ & $3 \cdot 31$ & & \\
\hline
\end{tabular}

Table 2. Torque index (TOR) at $\mathrm{T} 0$ and $\mathrm{Tl}$ : Student's $t$-test results

\begin{tabular}{lll}
\hline & TOR_T0 v. TOR_T1 & TOR_Tl $_{\text {wo }}$ v. TOR_Tl \\
\hline Group F & $0 \cdot 0003^{* * *}$ & $0 \cdot 023^{*}$ \\
Group S & n.s. & n.s. \\
Group C & n.s & \\
\hline
\end{tabular}

$* * *$ Very significant.

*Significant.

unchanged after 12 months of therapy with the Michigan occlusal splint.

The control group had normal values of the torque index at $\mathrm{T} 0$ and $\mathrm{Tl}_{\mathrm{wo}}$.

The inter-group analysis showed a highly significant difference when $\mathrm{F}$ group and $\mathrm{C}$ group or $\mathrm{F}$ group and $\mathrm{S}$ group are compared at T0 $(P<0 \cdot 0001)$ (Table 3$)$.

\section{Discussion}

In healthy subjects, a mandibular rotation on the horizontal axis may be counterbalanced by the actions

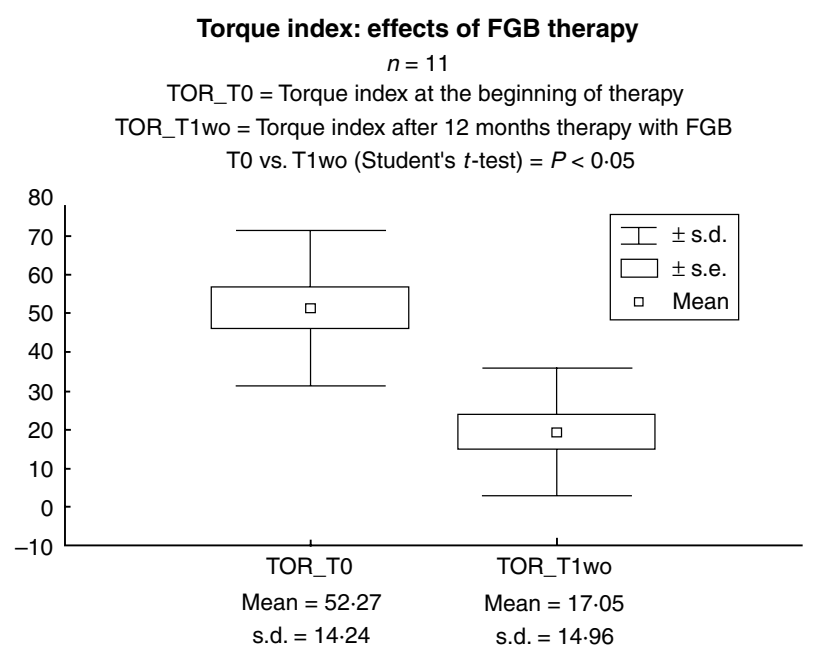

Fig. 2. MVC in intercuspal position: torque index at the beginning of therapy (TOR_T0) and after 12 months of therapy with FGB (TOR_T $1_{\text {wo }}$ ).

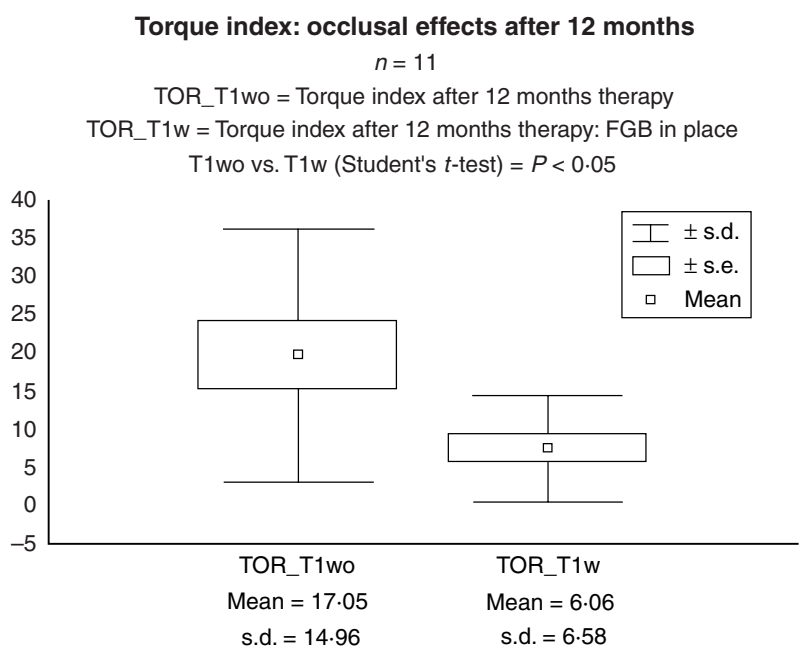

Fig. 3. MVC in intercuspal position after 12 months of therapy determined without FGB (TOR_Tl $1_{\text {wo }}$ ) and with FGB in place (TOR_Tl $1_{\mathrm{w}}$ ).

of ligaments and other jaw muscles forces. Probably the lack of this dynamic balance could be an etiologic factor in TMD. It has been demonstrated that facial asymmetry is of significant incidence in patients with TMD (22). Inui et al. (23) reported that facial asymmetry, if present, was characterized as a mandibular lateral displacement and a canted frontal occlusal plane ascending towards the displaced side of the mandible in TMD patients.

It has been reported that a reversible approach (drugs or splints) is preferable to minimize the risk of iatrogenic complications and because radical treatments are not superior to a reversible approach (24). The orthodonthic treatment of TMD patients is considered to be radical and to risk iatrogenic complications: there is a significant risk of failure. It is clear that there is no single approach to the management of TMD and the rationale underlying orthodontic treatment of TMD patients must be verified. Previous studies report some specific types of malocclusion (especially distal molar occlusion, Angle class III, open bite, extreme maxillary overjet, unilateral cross-bite and deep bite) to be significantly associated with signs and symptoms of $\operatorname{TMD}(5-8,25)$. The orthodontic correction of these occlusal features in children may reveal a reduction in signs and symptoms of TMD. An important issue is whether orthodontic therapy aimed at correcting the occlusion will affect the neuromuscular system. The torque index proposed by Ferrario et al. (21) may be helpful in these studies because it indicates the presence of resultant muscular forces with a laterodeviating effect on the mandible. 
Table 3. One-way ANova results: the differences of the torque index between the groups

\begin{tabular}{|c|c|c|c|c|c|c|c|c|c|}
\hline & \multicolumn{3}{|l|}{ T0 } & \multicolumn{3}{|l|}{$\mathrm{Tl}_{\text {wo }}$} & \multicolumn{3}{|l|}{$\mathrm{T} 1_{\mathrm{w}}$} \\
\hline & Mean difference & $q$-value & $P$-value & Mean difference & $q$-value & $P$-value & Mean difference & $q$-value & $P$-value \\
\hline C v. F & $-46 \cdot 66$ & $16 \cdot 018$ & $<0 \cdot 0001$ & $-11 \cdot 62$ & $3 \cdot 321$ & n.s. & -0.63 & $0 \cdot 244$ & n.s \\
\hline C v. S & $-4 \cdot 92$ & $1 \cdot 692$ & n.s. & $-7 \cdot 50$ & $2 \cdot 142$ & n.s. & $-4 \cdot 52$ & $1 \cdot 739$ & n.s \\
\hline F v. S & $41 \cdot 73$ & $14 \cdot 680$ & $<0.0001$ & $4 \cdot 1$ & $1 \cdot 208$ & n.s. & $-3 \cdot 89$ & 1.532 & n.s \\
\hline
\end{tabular}

The results of this study showed that FGB-D is able to correct an abnormal torque index after 12 months of therapy.

Blanskma and Van Eijden (26) reported that the anterior temporalis muscle is more concerned with laterodeviation of the mandible than with a closing action. Our data suggest that the FGB-D probably has more effect on the anterior temporalis muscle. This may be due to the stimulus from the periodontal mechanoceptors, but considering the change in jaw position, it may also be the result of a differential output from other peripheral sensory receptors, such as joint receptors and muscle spindles.

The group $S$ showed a close to normal torque index before and after therapy with the Michigan occlusal splint, and similar results were obtained from the control group. These data suggest that dental deep bite may be involved in some muscle-related TMD. Further investigation is necessary before we can fully understand the mechanism of its involvement.

\section{Conclusions}

The main conclusions of this study are:

(1) Quantitative sEMG of masticatory muscles cannot distinguish healthy subjects from those with musclerelated TMD (group $\mathrm{H}$ versus group $\mathrm{S}$ );

(2) Deep bite seems to be related to a high torque index and then to a lateral displacement of the mandible;

(3) The FGB-D reduces the torque index.

These findings suggest that FGB-D may be useful in muscle-related TMD patients requiring orthodontic treatment for dental deep bite correction. Because of the small number of patients investigated, these results can be considered to be preliminary.

\section{References}

1. Dworkin SF, Le Resche L. Research diagnostic criteria for temporomandibular disorders: review, criteria, examinations and specifications, critique. J Craniomand disord. 1992;6:302355.

2. Stohler CS. Muscle-related temporomandibular disorders. J Orofac Pain. 1999;13:273-284.

3. Bracco P, Deregibus A, Icardi K, Castroflorio T. Reliability of EMG. In: 12th ICCMO International Congress on Cranio-Mandibular Disorders, 'State of the Art' in Research and Practice. Frankfurt-Bad Homburg: ICCMO Publishers; 4-7 October 2001: 52.

4. Ferrario VF, Sforza C, Tartaglia GM, Dellavia C. Immediate effect of a stabilization split on masticatory muscle activity in temporomandibular disorders patients. J Oral Rehabil. 2002;29:810-815.

5. Tanne K, Tanaka E, Sakuda M. Association between malocclusion and temporomandibular disorders in orthodontic patients before treatment. J Orofac Pain. 1993;7:156-162.

6. Sonnesen L, Bakke M, Solow B. Malocclusion traits and symptoms and signs of temporomandibular disorders in children with severe malocclusion. Eur J Orthod. 1998;20:543-559.

7. Pullinger AG, Seligman DA. Quantification and validation of predictive values of occlusal variables in temporomandibular disorders using a multifactorial analysis. J Prosthet Dent. 2000;83:66-75.

8. Thilander B, Rubio G, Pena L, De Mayorga C. Prevalence of temporomandibular disorders and its association with malocclusion in children and adolescents: an epidemiologic study related to specific stages of dental development. Angle Orthod. 2002;72:146-154.

9. Bracco P, Solinas GF. Orthognatic correction of uni- and bilateral crossbite with a functional appliance. Mondo Ortod. 1979;4:8-24.

10. Sessle BJ, Woodside DG, Bourque P et al. Effect of functional appliances on jaw muscle activity. Am J Orthod and Dentofacial Orthop. 1990;98:222-230.

11. Yamin-Lacouture C, Woodside DG, Sectakof PA, Sessle BJ. The action of three types of functional appliances on the activity of the masticatory muscles. Am J Orthod and Dentofacial Orthop. 1997;112:560-572.

12. Tallgren A, Christiansen RL, Ash M Jr, Miller RL. Effects of a myofunctional appliance on orofacial muscles and structures. Angle Orthod. 1998;68:249-258.

13. Bracco P, Solinas GF. Use and control of the 'functional bite plate' in the early treatment of crossbite. Mondo Ortod. 1979;4:7-17.

14. Bracco P, Deregibus A, Viora E. An original functional appliance for the correction of open-bite and crossbite. In: 
95th American Association of Orthodontics Annual Session and 4th International Orthodontic Congress. San Francisco, CA, 12-17 May 1995.

15. Bracco P, Deregibus A, Viora E. Anteroposterior evaluation of crossbite correction using an original functional appliance. In: 96th American Association of Orthodontics Annual Session. Denver, CO, 11-15 May 1996.

16. Bracco P, Deregibus A, Viora E. Dental and structural crossbite correction using an original functional appliance. In: 98th American Association of Orthodontics Annual Session. Dallas, TX, 15-20, May 1998.

17. Ash MM, Ramfjord SP. An Introduction to Functional Occlusion. Philadelphia, PA: W.B. Saunders; 1982.

18. Iwasaki S, Tokunaga T, Baba S, Tanaka M, Kawazoe T. Non invasive location of the end plate in the human masseter muscle using surface electromyography with an electrode array. J Osaka Dent Univ. 1990;24:135-140.

19. Tokunaga T, Baba S, Tanaka M, Kashiwagi K, Kimura K, Kawazoe T. Two-dimensional configuration of the myoneural junctions of human masticatory muscle detected with matrix electrode. J Oral Rehabil. 1998;25:329-334.

20. Ferrario VF, Sforza C, Colombo A, Ciusa V. An electromyographic investigation of masticatory muscles symmetry in normo-occlusion subjects. J Oral Rehabil. 2000;27:33-40.
21. Ferrario VF, Sforza C, Miani A, Jr, D’Addona A, Barbini E. Electromyographic activity of human masticatory muscles in normal young people. Statistical evaluation of reference values for clinical applications. J Oral Rehabil. 1993;20:271280.

22. Inui M, Fushima K, Sato S. Facial asymmetry in temporomandibular joint disorders. J Oral Rehabil. 1999;26: 402-406.

23. Inui $M$, Fushima $K$, Sato S. Dental asymmetry in temporomandibular disorders. J Oral Rehabil. 1999;26:752-756.

24. Stohler CS, Zarb GA. On the management of temporomandibular disorders: a plea for a low-tech, high prudence therapeutic approach. J Orofac Pain. 1999;13:255-261.

25. McNamara JA Jr, Seligman DA, Okeson JP. Occlusion, orthodontic treatment, and temporomandibular disorders: a review. J Orofac Pain. 1995; 9:73-90.

26. Blanskma NG, van Eijden TMGJ. Electromyographic heterogeneity in the human temporalis and masseter muscles during static biting, open/close excursions, and chewing. J Dent Res. 1995;74:1318-1327.

Correspondence: Tommaso Castroflorio, Via M. Coppino 106, 10147 Torino, Italia.

E-mail: tcastroflorio@libero.it 